\title{
Progression of Aortic Stenosis in Patients with Bicuspid Aortic Valve
}

\author{
Michael Shang ${ }^{1}$, Arianna Kahler-Quesada ${ }^{1}$, Makoto Mori ${ }^{2}$, Sameh Yousef ${ }^{1}$, Arnar \\ Geirsson $^{2}$, and Prashanth Vallabhajosyula ${ }^{1}$ \\ ${ }^{1}$ Yale School of Medicine \\ ${ }^{2}$ Yale University School of Medicine
}

August 5, 2021

\begin{abstract}
Background: Bicuspid aortic valve is the most common congenital heart defect and predisposes patients to developing aortic stenosis more frequently and at a younger age than the general population. However, the influence of bicuspid aortic valve on the rate of progression of aortic stenosis remains unclear. Methods: In 236 patients ( 177 tricuspid aortic valve, 59 bicuspid aortic valve) matched by initial severity of mild or moderate aortic stenosis, we retrospectively analyzed baseline echocardiogram at diagnosis with latest available follow-up echocardiogram. Baseline comorbidities, annualized progression rate of hemodynamic parameters, and hazard of aortic valve replacement were compared between valve phenotypes. Results: Median echocardiographic follow-up was 2.6 (IQR 1.6-4.2) years. Patients with tricuspid aortic stenosis were significantly older with more frequent comorbid hypertension and congestive heart failure. Median annualized progression rate of mean gradient was 2.3 (IQR 0.6-5.0) $\mathrm{mmHg} /$ year vs. 1.5 (IQR 0.5-4.1) $\mathrm{mmHg} /$ year $(\mathrm{p}=0.5$ ), and that of peak velocity was 0.14 (IQR $0-0.31$ ) $\mathrm{m} / \mathrm{s} /$ year vs. 0.10 (IQR 0.04-0.26) $\mathrm{m} / \mathrm{s} /$ year $(\mathrm{p}=0.7)$ for tricuspid vs. bicuspid aortic valve, respectively. On multivariate analyses, bicuspid aortic valve was not significantly associated with more rapid progression of aortic stenosis. In a stepwise Cox proportional hazards model adjusted for baseline mean gradient, bicuspid aortic valve was associated with increased hazard of aortic valve replacement (HR: 1.7, 95\% CI [1.0, 3.0], p=0.049). Conclusion: Bicuspid aortic valve may not significantly predispose patients to more rapid progression of mild or moderate aortic stenosis. Guidelines for echocardiographic surveillance of aortic stenosis need not be influenced by valve phenotype.
\end{abstract}

\section{Progression of Aortic Stenosis in Patients with Bicuspid Aortic Valve}

Running head: Progression of bicuspid aortic stenosis

Michael Shang BS, ${ }^{1}$ Arianna Kahler-Quesada BS, ${ }^{1}$ Makoto Mori MD, 1 Sameh Yousef MD, ${ }^{1}$ Arnar Geirsson $\mathrm{MD},{ }^{1}$ Prashanth Vallabhajosyula $\mathrm{MD}^{1}$

Division of Cardiac Surgery, Yale University School of Medicine, New Haven, CT

Sources of Fundings: No funding sources were used for this study.

Corresponding Author:

Prashanth Vallabhajosyula, MD, MS

Associate Professor of Surgery

Division of Cardiac Surgery

Yale School of Medicine 
330 Cedar Street, Boardman Building 204

New Haven, CT 06520-8062

Email: prashanth.vallabhajosyula@yale.edu

Phone: 203-785-5000

\section{Abstract}

Background: Bicuspid aortic valve is the most common congenital heart defect and predisposes patients to developing aortic stenosis more frequently and at a younger age than the general population. However, the influence of bicuspid aortic valve on the rate of progression of aortic stenosis remains unclear.

Methods: In 236 patients (177 tricuspid aortic valve, 59 bicuspid aortic valve) matched by initial severity of mild or moderate aortic stenosis, we retrospectively analyzed baseline echocardiogram at diagnosis with latest available follow-up echocardiogram. Baseline comorbidities, annualized progression rate of hemodynamic parameters, and hazard of aortic valve replacement were compared between valve phenotypes.

Results: Median echocardiographic follow-up was 2.6 (IQR 1.6-4.2) years. Patients with tricuspid aortic stenosis were significantly older with more frequent comorbid hypertension and congestive heart failure. Median annualized progression rate of mean gradient was 2.3 (IQR 0.6-5.0) $\mathrm{mmHg} /$ year vs. 1.5 (IQR 0.54.1) $\mathrm{mmHg} /$ year $(\mathrm{p}=0.5)$, and that of peak velocity was 0.14 (IQR 0-0.31) $\mathrm{m} / \mathrm{s} /$ year vs. 0.10 (IQR 0.04-0.26) $\mathrm{m} / \mathrm{s} /$ year $(\mathrm{p}=0.7)$ for tricuspid vs. bicuspid aortic valve, respectively. On multivariate analyses, bicuspid aortic valve was not significantly associated with more rapid progression of aortic stenosis. In a stepwise Cox proportional hazards model adjusted for baseline mean gradient, bicuspid aortic valve was associated with increased hazard of aortic valve replacement (HR: 1.7, 95\% CI [1.0, 3.0], p=0.049).

Conclusion: Bicuspid aortic valve may not significantly predispose patients to more rapid progression of mild or moderate aortic stenosis. Guidelines for echocardiographic surveillance of aortic stenosis need not be influenced by valve phenotype.

Text

\section{Introduction}

Aortic stenosis (AS) is a common cause of valvular heart disease worldwide. Its prevalence increases with age, ranging from $4.6 \%-6.4 \%$ in U.S. adults aged 75 years and older. ${ }^{1,2}$ The most common etiology of aortic stenosis is degenerative calcification, which is primarily associated with aging as well as other cardiovascular disease. However, the presence of bicuspid aortic valve (BAV) represents a major risk factor in the development of AS in younger populations and is present in $65 \%$ of adults aged 60 and younger undergoing aortic valve replacement for aortic stenosis. ${ }^{3}$ While patients with BAV are known to develop AS more frequently and earlier than patients with tricuspid aortic valve (TAV), it remains uncertain whether BAV is associated with faster progression of AS severity once AS develops, which is important to guide screening and intervention practices. ${ }^{4}$

While the progression rate of AS varies widely among individuals, older age, male sex, coronary artery disease, plasma levels of oxidized phospholipids, and baseline hemodynamic severity have all been associated with more rapid progression. ${ }^{5-8}$ BAV has been implicated in certain studies, ${ }^{9,}{ }^{10}$ but others have shown no difference in AS progression rate between BAV and TAV phenotypes. ${ }^{5,11}$ Furthermore, these findings were drawn from post hoc analyses of clinical trials aimed at determining effects of metabolic syndrome and plasma lipids on AS disease progression. As a result, these studies examined very small cohorts of BAV patients $(\mathrm{N}<41)$, representing a significant limitation in the current literature. These findings attest to the need for more investigations to better understand progression of more-than-mild AS in BAV patients.

Using a healthcare system-wide echocardiographic database, we aim to determine the hemodynamic progression rate of mild and moderate severe aortic stenosis in patients with BAV as compared to patients with TAV. 


\section{Materials and Methods}

\section{Study Population}

A total of 236 patients (59 BAV and 177 TAV) were selected from our health system-wide database of 60,383 echocardiography reports from 2013-2018. These included echocardiograms obtained for any indication at 32 sites encompassing 5 tertiary-care hospitals and over 120 clinics at satellite locations throughout the state. For the BAV cohort, the database was queried for all mentions of "bicuspid aortic valve" or "BAV". Initially, 222 patients with BAV confirmed by echocardiography were identified. We then reviewed the records of these patients and included patients with an initial diagnosis of mild or moderate AS and echocardiographic follow-up greater than or equal to 6 months from initial diagnosis of AS. Patients were excluded if they never progressed beyond "trace" severity or if they had an initial severity of "severe". Other exclusion criteria were active atrial fibrillation, active endocarditis, and history of aortic valve or aortic surgery prior to the initial echocardiogram. Ultimately, 59 patients with BAV were included in this study.

To identify our TAV cohort, we randomly sampled from our echo database for reports of mild and moderate AS in proportions identical to that of the initial BAV cohort. For each patient selected, we reviewed the medical record and enrolled them based on the criteria described above. Sampling continued until the cohort consisted of 177 TAV patients, stratified by mild or moderate initial severity in a 3:1 TAV-to-BAV ratio.

This study was approved by the Yale Institutional Review Board and written informed consent was waived.

\section{Clinical Data}

Comorbidity data were obtained by extracting ICD-10 data from each patient's medical record and converted using the Elixhauser Comorbidity Index. ${ }^{12}$ Patient demographic information, surgical history, and mortality data up to November 1, 2020 was obtained by review of the medical record.

\section{Echocardiography}

Echocardiographic data were drawn from complete echocardiograms including 2D, M-mode, and color doppler. Peak velocity was calculated by continuous wave doppler, mean gradient was calculated by averaging instantaneous pressure gradients converted from measured velocities using the Bernoulli equation, and aortic valve area (AVA) was calculated by the continuity equation. Biplane Simpson's technique was used to estimate ejection fraction (EF). Aortic valve phenotype was assessed in the parasternal short-axis view. Severity of aortic stenosis was assessed using established criteria for mean gradient, peak velocity, and aortic valve area. Though the echocardiography reports used to identify patients were only obtained from exams occurring between 2013-2018, we reviewed the electronic medical record for the first and last available echocardiograms between the period of January 1, 2005 and November 1, 2020. These were used to calculate the yearly progression rate of mean gradient $(\mathrm{mmHg} /$ year $)$ and peak velocity $(\mathrm{m} / \mathrm{s} /$ year$)$.

\section{Statistical Analysis}

Continuous variables were assessed using median (interquartile range [IQR]), and categorical variables are presented as N (\%). Wilcoxon rank-sum test was used to compare differences in continuous variables between BAV and TAV. Chi-square test and Fisher's exact test were used for categorical variables, as appropriate.

To determine risk factors associated with rapid progression of AS, we dichotomized patients based on progression rate of mean gradient greater than (rapid progressor) or less than or equal to (slow progressor) the average annualized progression of mean gradient for the whole cohort, as previously described. ${ }^{10,}{ }^{13}$ Univariate and multivariable logistic regression analysis was used to determine whether BAV is associated with rapid progression of AS. Variables with $\mathrm{p}<0.10$ on univariate analysis were included in the multivariate model. Kaplan-Meier curves were generated to compare BAV and TAV patients for occurrence of surgical or transcatheter aortic valve replacement (AVR) after initial diagnosis of AS. Stepwise multivariable Cox proportional hazards model was used to assess the association of BAV with AVR, adjusting for potential confounders. P-values $<0.05$ were considered statistically significant. All statistical analyses were performed using RStudio, version 1.2.1335 (Boston, MA, USA). 


\section{Results}

\section{Baseline Patient Characteristics}

A total of 236 patients with mild or moderate AS were included in this analysis. The BAV cohort consisted of 59 patients, of which 40 had an initial AS severity of mild (68\%) and 19 patients had moderate initial severity $(32 \%)$. The TAV cohort consisted of 177 patients, matched by initial severity with our BAV cohort (120 mild AS, 57 moderate AS). Of the 236 patients, 136 (58\%) were male and 199 (84\%) were Caucasian, without significant differences in sex and race between BAV and TAV cohorts. The BAV cohort was significantly younger than the TAV cohort (59 years vs. 78 years, $\mathrm{p}<.001$ ) and had a longer median echocardiography follow-up period ( 3.17 years vs. 2.40 years, $\mathrm{p}=0.02)$. The BAV cohort also had lower baseline prevalence of hypertension ( $69 \%$ vs. $82 \%, \mathrm{p}=0.043)$ and congestive heart failure $(6.8 \%$ vs. $28 \%, \mathrm{p}<0.001)$ than the TAV cohort. There were no significant differences between the two groups in terms of tobacco use, body mass index, pulmonary disease, chronic kidney disease, or peripheral vascular disease. Table 1 summarizes these demographics and comorbidities.

Baseline hemodynamics were similar between BAV and TAV groups (Table 2 ). Among all patients, mean gradient was 17.0 (IQR 13.0-21.1) $\mathrm{mmHg}$, peak velocity was 2.8 (IQR 2.5-3.1) $\mathrm{m} / \mathrm{s}$, and indexed aortic valve area was 0.71 (IQR $0.59-0.86) \mathrm{cm}^{2} / \mathrm{m}^{2}$.

\section{Hemodynamic Progression of $A S$}

Progression of hemodynamic parameters is shown in Table 2. Median echocardiographic interval was 2.6 (IQR 1.6-4.2) years and was significantly longer in the BAV cohort $(\mathrm{p}=0.011)$. Despite longer echocardiographic follow-up, the proportion of BAV patients who had progressed beyond initial baseline severity was similar to TAV patients ( $56 \%$ and $55 \%$, respectively, $\mathrm{p}=0.9)$. However, at latest follow-up, $22(37 \%) \mathrm{BAV}$ patients had developed severe AS compared to 50 (28\%) TAV patients ( $\mathrm{p}=0.062)$. Annualized progression rate was similar between groups, regardless of hemodynamic parameter used (Figure 1 ). Median annualized progression of mean gradient was 2.3 (IQR 0.6-5.0) $\mathrm{mmHg} /$ year for patients with TAV vs. 1.5 (IQR $0.5-4.1) \mathrm{mmHg} /$ year for patients with BAV $(\mathrm{p}=0.5)$. Median annualized progression of peak velocity was 0.14 (IQR $0-0.31) \mathrm{m} / \mathrm{s} /$ year for patients with TAV vs. 0.10 (IQR 0.04-0.26) $\mathrm{m} / \mathrm{s} /$ year in patients with BAV $(\mathrm{p}=0.4)$.

Patients were dichotomized into slow and rapid progressors based on the overall average annualized progression of mean gradient of $2.85 \pm 5.31 \mathrm{mmHg}$ /year. The proportion of rapid progressors was similar between cohorts, with $74(43 \%)$ rapid progressors in the TAV group and $21(36 \%)$ in the BAV group (p=0.4), Table 2 .

\section{Factors Associated with Faster Hemodynamic Progression of Aortic Stenosis}

On univariate analysis, there was no significant difference between aortic valve morphology and rapid progressor phenotype (Table 3 ). Factors that were significantly associated with rapid progression were age (OR: 1.02, 95\% CI [1.00, 1.04]; $\mathrm{p}=0.03$ ) and baseline mean gradient (OR: 1.04, 95\% CI [1.00, 1.08]; $\mathrm{p}=0.04$ ). On the other hand, higher BMI was associated with slow progression (OR 0.95, 95\% CI [0.91, 0.99]; $\mathrm{p}=0.02$ ). When comparing comorbidities between the cohorts, BMI and presence of diabetes mellitus, chronic kidney disease, or hypertension was not significant between rapid and slow progressors and did not play a role in the progression of mean aortic gradient. On multivariate analysis adjusted for age, BMI, and baseline mean gradient, BAV was not significantly associated with rapid progression, $\mathrm{p}=0.7$ (Table 3 ).

\section{Association of Valve Phenotype with Aortic Valve Replacement}

Median clinical follow-up time was similar between groups (TAV: 6.2 (IQR 1.7-10.6) years vs. BAV: 6.4 (IQR 3.4-9.4) years, $\mathrm{p}=0.43)$. During the study period, $33(19 \%)$ TAV patients and $22(37 \%)$ BAV patients underwent surgical or transcatheter AVR (Figure 2 ). However, median time to AVR after initial diagnosis of AS was similar between cohorts at 2.7 (IQR 2.1-5.5) years in TAV patients and 3.3 (2.3-4.8) years in BAV patients $(\mathrm{p}=0.09)$. A stepwise Cox proportional hazards model revealed that BAV (HR: 1.73, 95\% CI 
$[1.00,2.99], \mathrm{p}=0.049)$ and baseline mean gradient (HR: 1.07, 95\% CI [1.04, 1.10], $\mathrm{p}<0.001)$ were significantly associated with increased hazard of AVR (Table 4 ).

\section{Comment}

In this retrospective study of a matched cohort, we demonstrate that presence of BAV was not statistically significantly associated with more rapid hemodynamic progression of mild or moderate aortic stenosis. Despite this, patients with BAV were more likely to undergo AVR.

It is well-established that BAV patients develop AS more frequently and at an earlier age compared to the general population. This is reflected in our study in the significantly younger age of the BAV cohort. However, there is discordance in the literature about whether BAV is associated with faster progression of AS, once established. Two studies have found no association between valve morphology and annual progression rate of mean transvalvular gradient, even after adjusting for age.5, 11 Conversely, Ryu et al. and Shen et al. both report that after adjusting for age and baseline hemodynamic measurements, BAV is independently associated with faster annual progression. ${ }^{9}{ }^{10}$ However, in both the above studies, the BAV patients differed significantly from TAV patients in terms of baseline AS grade and hemodynamic parameters. It is now wellestablished that initial severity is an important predictor of more rapid progression. ${ }^{5,11,14}$ As a result, the confounding effect on progression rate introduced by differences in baseline severity between groups may bias the comparison.

The present study is novel in that we analyzed a larger cohort of BAV patients than prior studies, and we matched our BAV and TAV patients by initial AS grade. After doing so, we found no statistically significant association between aortic valve phenotype and unadjusted progression rate of mean gradient and peak velocity. Furthermore, risk of rapid progression was similar between groups, even after adjusting for comorbidities. In concordance with prior studies, older age and baseline severity were associated with more rapid progression.

Several cellular mechanisms have been proposed for the development and progression of AS. Regardless of valve phenotype, AS progression is thought to share similar pathways with atherosclerosis. Namely, oxidized lipid accumulation, inflammatory stimulation, and shear stress promote osteogenic differentiation of valvular interstitial cells (i.e. fibroblasts and smooth muscle cells). ${ }^{15-18}$ After induction of an osteoblast-like phenotype, valvular interstitial cells then upregulate calcium production, via BMP2 and matrix metalloproteinases, leading to calcification and stenosis. Several clinical studies have reported that these same cardiometabolic factors driving development of AS also lead to more rapid hemodynamic progression and calcification. ${ }^{19-21}$ However, in patients with BAV, the accelerated development of AS is driven by increased shear stress due to abnormal valve morphology, as well as by genetic mutations resulting in downregulation of the NOTCH1 gene, which is important in preventing osteogenic differentiation. ${ }^{22}$ While the pathogenesis of AS in BAV versus TAV is driven by separate processes, our findings suggest that this distinction does not result in clinically significant differences in rate of progression.

Despite this, we observed that over similar follow-up periods, BAV patients had shorter time to AVR (surgical or transcatheter) following diagnosis of mild or moderate AS, after adjusting for baseline mean transvalvular gradient. We were unable to fully account for the complex factors involved in preoperative decision making for these patients, which limits our ability to adequately determine differences in timing of intervention. Nevertheless, the decreased hazard of intervention in TAV patients could be driven by their older age or perceived operative risk, as shown in some studies of elderly patients who were not operated on despite severe symptomatic AS. ${ }^{23}, 24$ Additionally, BAV patients are at increased risk for developing thoracic aortic aneurysms, and present for aneurysm surgery more frequently than TAV patients. ${ }^{25}$ This may reflect the surgeon's lower threshold for replacing the aortic valve during concomitant aortic surgery; however, differences in AS severity and symptoms at time of surgery in BAV vs. TAV has not been reported in the literature. While BAV patients develop AS earlier in life, surveillance patterns and threshold for procedural intervention should not be driven by phenotype alone. Rather, presence of atherosclerotic risk factors, baseline severity and clinically observed progression rate may be more accurate predictors of long-term outcome. 


\section{Limitations}

Due to the retrospective nature of this study, the hemodynamic data limited to what was documented in echocardiography reports. We did not evaluate interobserver variability by independent review of hemodynamic parameters. As such, we were unable to account for effects of BAV morphology (i.e. cusp fusion pattern, presence of raphe) as they were inconsistently reported on. In addition, this was a single center study, which limits generalizability and raises the possibility of not capturing outside imaging and operative records, though an extensive chart review was conducted using our electronic medical record. Lastly, the power of statistical conclusions drawn from this sample are limited by the sample size of the BAV cohort. Our findings warrant future multicenter studies in order to achieve larger a sample of BAV patients.

\section{Conclusion}

Overall, this study adds to the growing body of evidence to suggest that aortic stenosis in bicuspid aortic valves may not progress faster than that in tricuspid valves. While frequent monitoring according to current guidelines will continue to be crucial to the management of aortic stenosis, our data suggests that the severity of AS and observed progression rate are more predictive of future progression than valve phenotype.

\section{References}

1. Nkomo VT, Gardin JM, Skelton TN, Gottdiener JS, Scott CG and Enriquez-Sarano M. Burden of valvular heart diseases: a population-based study. Lancet . 2006;368:1005-11.

2. Stewart BF, Siscovick D, Lind BK, Gardin JM, Gottdiener JS, Smith VE, Kitzman DW and Otto CM. Clinical factors associated with calcific aortic valve disease. Cardiovascular Health Study. J Am Coll Cardiol . 1997;29:630-4.

3. Roberts WC and Ko JM. Frequency by decades of unicuspid, bicuspid, and tricuspid aortic valves in adults having isolated aortic valve replacement for aortic stenosis, with or without associated aortic regurgitation. Circulation . 2005;111:920-5.

4. Beppu S, Suzuki S, Matsuda H, Ohmori F, Nagata S and Miyatake K. Rapidity of progression of aortic stenosis in patients with congenital bicuspid aortic valves. Am J Cardiol . 1993;71:322-7.

5. Nguyen V, Cimadevilla C, Estellat C, Codogno I, Huart V, Benessiano J, Duval X, Pibarot P, Clavel MA, Enriquez-Sarano M, Vahanian A and Messika-Zeitoun D. Haemodynamic and anatomic progression of aortic stenosis. Heart . 2015;101:943-7.

6. Bahler RC, Desser DR, Finkelhor RS, Brener SJ and Youssefi M. Factors leading to progression of valvular aortic stenosis. Am J Cardiol . 1999;84:1044-8.

7. Rosenhek R, Klaar U, Schemper M, Scholten C, Heger M, Gabriel H, Binder T, Maurer G and Baumgartner H. Mild and moderate aortic stenosis. Natural history and risk stratification by echocardiography. Eur Heart J . 2004;25:199-205.

8. Capoulade R, Chan KL, Yeang C, Mathieu P, Bosse Y, Dumesnil JG, Tam JW, Teo KK, Mahmut A, Yang X, Witztum JL, Arsenault BJ, Despres JP, Pibarot P and Tsimikas S. Oxidized Phospholipids, Lipoprotein(a), and Progression of Calcific Aortic Valve Stenosis. J Am Coll Cardiol . 2015;66:1236-1246.

9. Shen M, Tastet L, Capoulade R, Arsenault M, Bedard E, Clavel MA and Pibarot P. Effect of bicuspid aortic valve phenotype on progression of aortic stenosis. Eur Heart J Cardiovasc Imaging . 2020;21:727-734.

10. Ryu DR, Park SJ, Han H, Lee HJ, Chang SA, Choi JO, Lee SC, Park SW and Oh JK. Progression rate of aortic valve stenosis in korean patients.J Cardiovasc Ultrasound . 2010;18:127-33.

11. Chan KL, Teo K, Dumesnil JG, Ni A, Tam J and Investigators A. Effect of Lipid lowering with rosuvastatin on progression of aortic stenosis: results of the aortic stenosis progression observation: measuring effects of rosuvastatin (ASTRONOMER) trial. Circulation . 2010;121:306-14. 
12. Quan H, Sundararajan V, Halfon P, Fong A, Burnand B, Luthi JC, Saunders LD, Beck CA, Feasby TE and Ghali WA. Coding algorithms for defining comorbidities in ICD-9-CM and ICD-10 administrative data.Med Care . 2005;43:1130-9.

13. Palta S, Pai AM, Gill KS and Pai RG. New insights into the progression of aortic stenosis: implications for secondary prevention. Circulation . 2000;101:2497-502.

14. Kim D, Chae D, Shim CY, Cho IJ, Hong GR, Park K and Ha JW. Predicting Disease Progression in Patients with Bicuspid Aortic Stenosis Using Mathematical Modeling. J Clin Med . 2019;8.

15. Abdelbaky A, Corsini E, Figueroa AL, Subramanian S, Fontanez S, Emami H, Hoffmann U, Narula $\mathrm{J}$ and Tawakol A. Early aortic valve inflammation precedes calcification: a longitudinal FDG-PET/CT study.Atherosclerosis . 2015;238:165-72.

16. O'Brien KD, Reichenbach DD, Marcovina SM, Kuusisto J, Alpers CE and Otto CM. Apolipoproteins $\mathrm{B}$, (a), and $\mathrm{E}$ accumulate in the morphologically early lesion of 'degenerative' valvular aortic stenosis.Arterioscler Thromb Vasc Biol. 1996;16:523-32.

17. Venardos N, Nadlonek NA, Zhan Q, Weyant MJ, Reece TB, Meng X and Fullerton DA. Aortic valve calcification is mediated by a differential response of aortic valve interstitial cells to inflammation. $J$ Surg Res . 2014;190:1-8.

18. Cote N, Mahmut A, Bosse Y, Couture C, Page S, Trahan S, Boulanger MC, Fournier D, Pibarot P and Mathieu P. Inflammation is associated with the remodeling of calcific aortic valve disease. Inflammation . 2013;36:573-81.

19. Tastet L, Capoulade R, Clavel MA, Larose E, Shen M, Dahou A, Arsenault M, Mathieu P, Bedard E, Dumesnil JG, Tremblay A, Bosse Y, Despres JP and Pibarot P. Systolic hypertension and progression of aortic valve calcification in patients with aortic stenosis: results from the PROGRESSA study. Eur Heart $J$ Cardiovasc Imaging . 2017;18:70-78.

20. Lindman BR, Clavel MA, Mathieu P, Iung B, Lancellotti P, Otto CM and Pibarot P. Calcific aortic stenosis. Nat Rev Dis Primers . 2016;2:16006.

21. Katz R, Wong ND, Kronmal R, Takasu J, Shavelle DM, Probstfield JL, Bertoni AG, Budoff MJ and O'Brien KD. Features of the metabolic syndrome and diabetes mellitus as predictors of aortic valve calcification in the Multi-Ethnic Study of Atherosclerosis. Circulation . 2006;113:2113-9.

22. Acharya A, Hans CP, Koenig SN, Nichols HA, Galindo CL, Garner HR, Merrill WH, Hinton RB and Garg V. Inhibitory role of Notch1 in calcific aortic valve disease. PLoS One . 2011;6:e27743.

23. Bach DS, Siao D, Girard SE, Duvernoy C, McCallister BD, Jr. and Gualano SK. Evaluation of patients with severe symptomatic aortic stenosis who do not undergo aortic valve replacement: the potential role of subjectively overestimated operative risk. Circ Cardiovasc Qual Outcomes . 2009;2:533-9.

24. Iung B, Cachier A, Baron G, Messika-Zeitoun D, Delahaye F, Tornos P, Gohlke-Barwolf C, Boersma E, Ravaud P and Vahanian A. Decision-making in elderly patients with severe aortic stenosis: why are so many denied surgery? Eur Heart J . 2005;26:2714-20.

25. Davies RR, Kaple RK, Mandapati D, Gallo A, Botta DM, Jr., Elefteriades JA and Coady MA. Natural history of ascending aortic aneurysms in the setting of an unreplaced bicuspid aortic valve.Ann Thorac Surg . 2007;83:1338-44.

\begin{tabular}{llll}
\hline & TAV, N=177 & BAV, N=59 & p-value \\
\hline $\begin{array}{l}\text { Age (years) } \\
\text { Race }\end{array}$ & $80(73,86)$ & $60(51,68)$ & $<\mathbf{0 . 0 0 1}$ \\
African American & $16(9.0 \%)$ & $8(14 \%)$ & 0.4
\end{tabular}




\begin{tabular}{|c|c|c|c|}
\hline & TAV, $\mathrm{N}=177$ & BAV, $\mathrm{N}=59$ & p-value \\
\hline Other & $9(5.1 \%)$ & $4(6.8 \%)$ & \\
\hline Caucasian & $152(86 \%)$ & $47(80 \%)$ & \\
\hline Sex & & & 0.11 \\
\hline Female & $81(46 \%)$ & $20(34 \%)$ & \\
\hline Male & $96(54 \%)$ & $39(66 \%)$ & \\
\hline Tobacco Use (years) & $28(17)$ & $28(18)$ & 0.7 \\
\hline Body Mass Index $\left(\mathrm{kg} / \mathrm{m}^{2}\right)$ & $28(7)$ & $29(6)$ & 0.12 \\
\hline Hypertension & $145(82 \%)$ & $41(69 \%)$ & 0.043 \\
\hline Pulmonary Disease & $43(24 \%)$ & $14(24 \%)$ & $>0.9$ \\
\hline Chronic Kidney Disease & $23(13 \%)$ & $9(15 \%)$ & 0.7 \\
\hline Diabetes Mellitus & $52(29 \%)$ & $13(22 \%)$ & 0.3 \\
\hline Peripheral Vascular Disease & $23(13 \%)$ & $11(19 \%)$ & 0.3 \\
\hline Congestive Heart Failure & $51(29 \%)$ & $4(6.8 \%)$ & $<0.001$ \\
\hline
\end{tabular}

Tables

Table 1: Patient Demographics and Comorbidities. Continuous variables are expressed as median (IQR) and categorical variables are expressed as N (\%). Statistically significant p-values are bolded.

BAV: bicuspid aortic valve, TAV: tricuspid aortic valve

\begin{tabular}{llll}
\hline & TAV, N=177 & BAV, N=59 & p-value \\
\hline $\begin{array}{l}\text { Median follow-up (years) } \\
\text { Baseline AS grade }\end{array}$ & $2.40(1.48-3.93)$ & $3.17(1.92-4.92)$ & $\mathbf{0 . 0 2}$ \\
Mild & $120(68 \%)$ & $40(68 \%)$ & $>0.9$ \\
Moderate & $57(32 \%)$ & $19(32 \%)$ & \\
AS grade at last follow-up & & & 0.062 \\
None & $8(4.5 \%)$ & $7(12 \%)$ & \\
Mild & $50(28 \%)$ & $15(25 \%)$ & \\
Moderate & $69(39 \%)$ & $15(25 \%)$ & \\
Severe & $50(28 \%)$ & $22(37 \%)$ & 0.2 \\
Baseline Mean Gradient (mmHg) & $16(13-20)$ & $18(14-22)$ & 0.5 \\
Baseline Peak Velocity (m/s) & $2.70(2.50-3.00)$ & $2.80(2.50-3.11)$ & 0.2 \\
Baseline Aortic Valve Area (cm2) & $1.30(1.10-1.60)$ & $1.35(1.18-1.70)$ & 0.2 \\
Baseline AVA Index (cm2/m2) & $0.72(0.59-0.86)$ & $0.70(0.60-0.82)$ & 0.7 \\
Progression Rate of Mean Gradient (mmHg/year) & $2.3(0.6-5.0)$ & $1.5(0.5-4.1)$ & 0.5 \\
Progression Rate of Peak Velocity (m/s/year) & $0.14(0.00-0.31)$ & $0.10(0.04-0.26)$ & 0.7 \\
Progression of AS grade & $105(59 \%)$ & $40(68 \%)$ & 0.2 \\
Rapid Progressors (>2.85mmHg/year) & $74(43 \%)$ & $21(36 \%)$ & 0.4 \\
\hline
\end{tabular}

Table 2: Baseline Echocardiography and Progression Rate of Aortic Stenosis. Continuous variables are expressed as median (IQR) and categorical variables are expressed as N (\%). Progression rate was calculated by dividing the difference in mean gradient or peak velocity between first and last echocardiograms by time elapsed between scans. Patients were determined to be rapid progressors if annualized progression of mean gradient was greater than the average of that for the entire study cohort.

AS: aortic stenosis, AVA: aortic valve area 


\begin{tabular}{llllllll}
\hline & Univariate & Univariate & Univariate & Univariate & Multivariate & Multivariate & Multi \\
\hline & $\mathbf{O R}$ & $\mathbf{9 5 \%}$ CI & $\mathbf{9 5 \%}$ CI & p-value & $\mathbf{O R}$ & $\mathbf{9 5 \%}$ CI & p-val \\
Age & 1.02 & {$[1.00,1.04]$} & {$[1.00,1.04]$} & $\mathbf{0 . 0 3 1}$ & 1.02 & {$[1.00,1.05]$} & 0.1 \\
Race & & & & & & & \\
African American & - & - & - & & & \\
Other & 0.19 & {$[0.01,1.28]$} & {$[0.01,1.28]$} & 0.14 & & \\
Caucasian & 1.84 & {$[0.75,4.97]$} & {$[0.75,4.97]$} & 0.2 & & & \\
Sex & & & & & & \\
Female & - & - & - & & & \\
Male & 1.31 & {$[0.77,2.25]$} & {$[0.77,2.25]$} & 0.3 & & \\
Tobacco Use (years) & 1 & {$[0.97,1.02]$} & {$[0.97,1.02]$} & 0.8 & & \\
Body Mass Index & 0.95 & {$[0.91,0.99]$} & {$[0.91,0.99]$} & $\mathbf{0 . 0 2}$ & 0.95 & & \\
Hypertension & 0.7 & {$[0.37,1.32]$} & {$[0.37,1.32]$} & 0.3 & & & \\
Pulmonary Disease & 1 & {$[0.54,1.83]$} & {$[0.54,1.83]$} & $>0.9$ & & & \\
Chronic Kidney Disease & 1.13 & {$[0.53,2.40]$} & {$[0.53,2.40]$} & 0.7 & & & \\
Diabetes Mellitus & 1.01 & {$[0.56,1.81]$} & {$[0.56,1.81]$} & $>0.9$ & & & \\
Peripheral Vascular Disease & 0.72 & {$[0.32,1.54]$} & {$[0.32,1.54]$} & 0.4 & & & \\
Congestive Heart Failure & 1.02 & {$[0.54,1.90]$} & {$[0.54,1.90]$} & $>0.9$ & & & \\
LV Ejection Fraction & 0.99 & {$[0.96,1.01]$} & {$[0.96,1.01]$} & 0.4 & & & \\
BAV & 0.76 & {$[0.41,1.39]$} & {$[0.41,1.39]$} & 0.4 & 1.14 & {$[0.51,2.55]$} & 0.7 \\
Baseline Mean Gradient & 1.04 & {$[1.00,1.08]$} & {$[1.00,1.08]$} & $\mathbf{0 . 0 3 7}$ & 1.05 & {$[1.01,1.10]$} & $\mathbf{0 . 0 0 8}$ \\
\hline
\end{tabular}

Table 3: Univariate and multivariate logistic regression for rapid progression of aortic stenosis. Rapid progression is defined as annualized progression of mean gradient greater than the average for the whole study cohort $(>2.85 \mathrm{mmHg} /$ year $)$. Statistically significant p-values are bolded.

BAV: bicuspid aortic valve, CI: confidence interval, LV: left ventricle, OR: odds ratio

\begin{tabular}{lllll}
\hline Covariate & $\boldsymbol{\beta}$ & HR & $\mathbf{9 5 \%}$ CI & p-value \\
\hline BAV & 0.55 & 1.73 & {$[1.00,2.99]$} & $\mathbf{0 . 0 4 9}$ \\
Baseline Mean Gradient & 0.068 & 1.07 & {$[1.04,1.10]$} & $<\mathbf{0 . 0 0 1}$ \\
\hline
\end{tabular}

Table 4: Stepwise Cox proportional hazards model for freedom from aortic valve replacement . Both surgical and transcatheter aortic valve replacement were included. Bicuspid aortic valve and baseline mean gradient were included in the model after stepwise adjustment.

BAV: bicuspid aortic valve, HR: hazard ratio, CI: confidence interval

Figure Legends

Figure 1: Annualized progression rate of peak velocity and mean gradient between TAV and BAV cohorts. (A ) Annualized progression of peak velocity ( $\mathrm{m} / \mathrm{s} /$ year). (B) Annualized progression of mean gradient $(\mathrm{mmHg} /$ year$)$. Box shows $25^{\text {th }}$ and $75^{\text {th }}$ percentiles, bolded line shows median value, and error bars show $10^{\text {th }}$ and $90^{\text {th }}$ percentiles. Median [interquartile range] are shown at the top of the graphs.

BAV: bicuspid aortic valve, TAV: tricuspid aortic valve

Figure 2: Kaplan-Meier curve for freedom from aortic valve replacement (surgical or transcatheter). Curve is truncated at 5 years. Censoring events include death and loss to follow-up. Shading represents $95 \%$ confidence interval. P-value was derived from log-rank test.

AVR: aortic valve replacement, BAV: bicuspid aortic valve, TAV: tricuspid aortic valve 

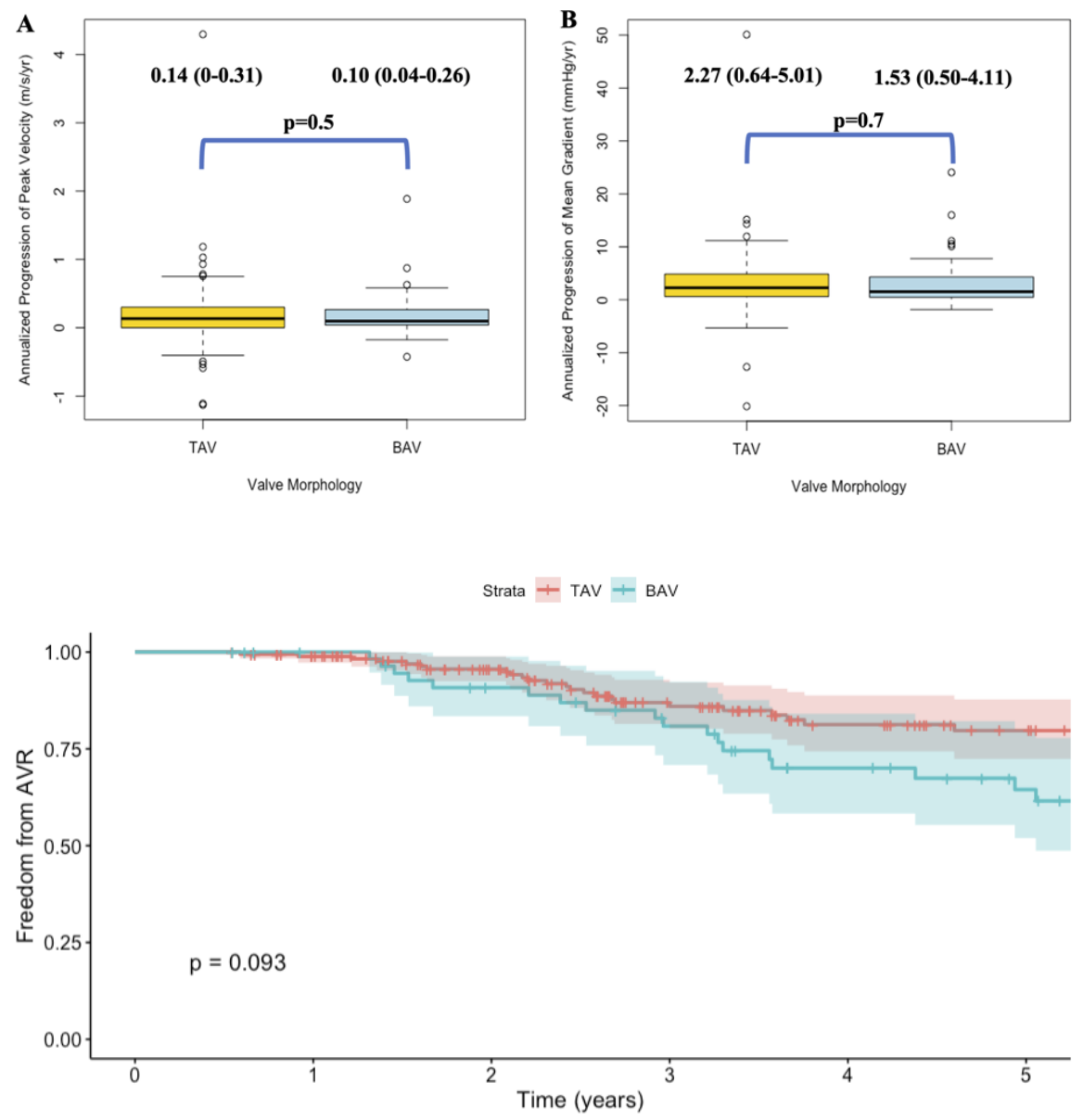

Number at risk: n (\%)

\begin{tabular}{|c|c|c|c|c|c|}
\hline$\frac{\mathbb{3}}{\mathbb{N}}$ TAV $-177(100)$ & $167(94)$ & $134(76)$ & $89(50)$ & $63(36)$ & $50(28)$ \\
\hline $59(100)$ & $55(93)$ & $47(80)$ & $39(66)$ & $29(49)$ & $22(37)$ \\
\hline 0 & 1 & 2 & s) ${ }^{3}$ & 4 & 5 \\
\hline
\end{tabular}

Cumulative number of events

\begin{tabular}{|c|c|c|c|c|c|c|}
\hline \multirow{3}{*}{$\begin{array}{l}\text { 丞 } \mathrm{TAV} \\
\text { क } \\
\text { BAV - }\end{array}$} & 0 & 2 & 7 & 19 & 23 & 24 \\
\hline & 0 & 0 & 5 & 10 & 15 & 17 \\
\hline & 0 & 1 & 2 & 3 & 4 & 5 \\
\hline
\end{tabular}

